\title{
Contribución al estudio léxico-semántico del verbo ponerse
}

\author{
MARÍA EUgenia CONDE NOGUEROL \\ Senior Lecturer/Lectora MAEC-AECID \\ University of Malaya \\ Facultad de Lenguas y Lingüística \\ 50603 Kuala Lumpur \\ E-mail: eugenia.noguerol@gmail.com
}

\section{CONTRIBUCIÓN AL ESTUDIO LÉ- XICO-SEMÁNTICO DEL VERBO PONERSE}

RESUMEN: Este trabajo es una contribución al estudio léxico-semántico del verbo ponerse como verbo de apoyo para la expresión de cambio de estado en español cuando este afecta a entidades animadas. A partir de su estudio combinatorio y de sus ocurrencias en el CREA, hemos podido agrupar los diferentes atributos con los que se combina y establecer clasificaciones semánticas bien delimitadas que, junto a otros criterios semántico-sintácticos, como la estructura actancial, la estructura sintáctica y el punto de vista o focalización, nos han permitido extraer conclusiones sobre el comportamiento real del verbo ponerse.

Debido a la complejidad aspectual que presenta, hemos tenido en cuenta aspectos de tipo semántico, sintáctico y contextual. Además, en algunas ocasiones hemos recurrido a factores de tipo pragmático que, si bien no tienen relevancia gramatical, han ayudado a una interpretación más exhaustiva.

Como veremos, ponerse, pues, es un verbo de apoyo que permite expresar cambios de estado que afectan al estado físico, al estado psíquico y al comportamiento o disposición humana.

PALABRAS CLAVES: ponerse; verbo de cambio; verbo de apoyo; semántica, clasificación verbal.

SUMARIO: Introducción. 2. Estudio de ponerse. 2.1 El cambio en entidades animadas. 2.1.1 Cambio de estado fisico. 2.1.1.1 Estado de salud. 2.1.1.2 Aspecto físico. 2.1.1.3 Color. 2.1.1.4 Postura corporal. 2.1.2 Cambio de estado psíquico. 2.1.2.1 Estado anímico. 2.1.3 Comportamientos o disposiciones humanas. 3 . Conclusiones.

\section{CONTRIBUTION TO THE LEXI- CAL-SEMANTIC STUDY OF THE VERB PONERSE}

ABSTRACT: This work is a contribution to the lexical-semantic study of the verb ponerse as a support verb for the expression in Spanish of a change of state affecting animated entities. From its combinatorial study and occurrences in the CREA, we have been able to group the different attributes with which this verb combines and establish well-defined semantic classifications that, together with other semantic-syntactic criteria, such as the actatial structure, the syntactic structure and the point of view or focus, have allowed us to draw conclusions about the actual behaviour of the verb ponerse.

Due its aspectual complexity, we have taken into account semantic, syntactic and contextual aspects. In addition, in some occasions we have resorted to pragmatic factors that, though having no grammatical relevance, have helped to obtain a more exhaustive interpretation. As we will see, ponerse is then a support verb that allows the expression of changes of state that affect the physical state, the psychic state and the human behaviour or disposition.

KEY WORDS: ponerse; verbs of change verb; support verb; semantics, verbal classification.

SUMMARY: 1 . Introduction. 2 . Study of ponerse. 2.1 The change in animated entities. 2.1.1 Change of physical state. 2.1.1.1 Health status. 2.1.1.2 Physical appearance. 2.1.1.3 Color. 2.1.1.4 Body posture. 2.1.2 Change of psychic state. 2.1.2.1 Mood state. 2.1.3 Human behaviors or dispositions. 3. Conclusions.

\section{CONTRIBUTION A L'ETUDE LEXICO-SEMANTIQUE DU VERBE PONERSE}

RÉSUMÉ: Ce travail est une contribution à l'étude lexicosémantique du verbe ponerse en tant que verbe de soutien à l'expression du changement d'état en espagnol lorsqu'il affecte des entités animées. A partir de son étude combinatoire et de ses occurrences dans le corpus CREA, nous avons pu regrouper les différents attributs avec lesquels il est combiné et établir des classifications sémantiques bien définies qui, avec d'autres critères sémantiques-syntaxiques, tels que la structure actantielle, la structure syntaxique et le point de vue ou la focalisation, nous ont permis de tirer des conclusions sur le comportement réel du verbe ponerse.

En raison de sa complexité aspectuelle, nous avons pris en compte des aspects, de type sémantique, syntaxiques et contextuels. En outre, nous avons parfois eu recours à des facteurs pragmatiques qui, bien que non pertinents sur le plan grammatical, ont contribué à donner une interprétation plus complète.

Comme nous le verrons, ponerse est donc un verbe qui permet d'exprimer des changements d'état qui affectent l'état physique, l'état psychique et le comportement ou la disposition humaine.

MOTS CLÉS: ponerse; verbe de changement; verbe de soutien; sémantique, classification verbale.

SOMMAIRE: 1. Introduction. 2 Étude de ponerse. 2.1 Le changement dans les entités animées. 2.1.1 Changement d'état physique. 2.1.1.1 État de santé. 2.1.1.2 Aspect physique. 2.1.1.3 Couleur. 2.1.1.4 Posture du corps. 2.1.2 Changement d'état psychique. 2.1.2.1 Etat d'humeur. 2.1.3 Comportements humains ou dispositions. 3. Conclusions.
Fecha de Recepción 


\title{
Contribución al estudio léxico-semántico del verbo ponerse
}

\author{
MARÍA EUgenia CONDE NOGUEROL
}

\section{INTRODUCCIÓN}

Este trabajo se perfila como una contribución al estudio léxico-semántico del verbo ponerse como verbo de apoyo $^{1}$ para la expresión el cambio de estado en español. Para ello, analizaremos sus posibles combinaciones tratando de explicar las peculiaridades semántico-contextuales que lo caracterizan $^{2}$.

Como verbo de apoyo funciona como herramienta para la expresión del cambio, y añade a la oración diversas informaciones, generalmente de tipo aspectual o modal, por lo que habrá que tener en cuenta su uso contextual para poder obtener un profundo análisis del mismo.

En primer lugar, se hace necesario establecer los criterios que hemos utilizado a la hora de delimitar tal unidad verbal. Debido a la complejidad aspectual que presenta, debemos tener en cuenta aspectos de tipo semántico, sintáctico y contextual. Asimismo, en algunas ocasiones hemos recurrido a factores de tipo pragmático que, si bien no tienen relevancia gramatical, pueden ayudar a una interpretación más exhaustiva.

Así, a la hora de enfrentarnos al análisis de este verbo, nos hemos valido de los siguientes criterios:

a) Punto de vista o focalización del proceso de cambio:

Partimos de la idea de que el cambio de estado o de entidad supone una situación compleja formada por un estado A (anterior al cambio), un umbral de cambio (proceso inicial del cambio) y un estado B final (después del cambio). Además, partimos de la idea de que una unidad verbal posee un determinado aspecto interno que permite destacar alguna parte, intervalo o faceta en la dinámica temporal interna del evento que describe; por ello, podemos distinguir entre aquellos verbos que focalizan el cambio en su inicio, en su final o resultado, o incluso, en el momento mismo del cambio. Consideramos adecuado este análisis porque creemos que el punto de vista o focalización se presenta como un rasgo pertinente o distintivo a la hora de describir los verbos semicopulativos de cambio, tales como hacerse, quedarse o volverse, y entre los que se encuentra ponerse.

\footnotetext{
${ }^{1}$ Son numerosos los trabajos sobre los verbos de apoyo en los que se incluyen algunos de los verbos de cambio como ponerse. Véase Moreno Burgos (2018), Gorp (2017), Conde Noguerol (2013), Morimoto y Pavón (2007) y Porroche (1988), entre otros.

2 Para ello nos hemos valido de los ejemplos extraídos del Corpus de Referencia del Español Actual (CREA), de la RAE.
} 


\section{b) Estructura actancial:}

Hemos tenido en cuenta, además, los componentes de la estructura actancial prototípica de estas unidad, asignándole a cada argumento una serie de propiedades ${ }^{3}$. En este sentido, la entidad afectada por el cambio, (A1), puede presentar los siguientes rasgos:

a. Tener el rasgo [+ / - animado].

b. Poseer [+ / - control] sobre el proceso de cambio.

c. Ejercer [+ / - esfuerzo] en la realización del cambio.

Por su parte, el elemento (A3) que se corresponde con el estado resultante del cambio, aquí representado por un adjetivo, un sustantivo o un sintagma, con la función de predicativo, denota la propiedad o característica adquirida por el objeto tras el cambio. Estas propiedades o características pueden clasificarse según designen:

a. Estados circunstanciales episódicos o cualidades caracterizadoras de personas o $\operatorname{cosas}^{4}$.

b. Adjetivos estativos o de sentido activo ${ }^{5}$.

Pueden poseer, asimismo, rasgos positivos o negativos y referirse a dimensiones de tipo físico o psíquico.

En cuanto al elemento (A2), esto es, la causa desencadenante del cambio, tendremos en cuenta si se trata de un ser animado, un ser inanimado o un suceso previo.

\section{c) Estructura sintáctica:}

Los diferentes actantes con que se combinan los verbos de cambio pueden presentar diversas funciones sintácticas, por lo que las tendremos también en cuenta en el estudio.

\footnotetext{
3 Para un análisis de las ventajas e inconvenientes de postular estructuras temáticas, vid. Bosque y Gutiérrez-Rexach (2009: 271-288).

4 Tomamos esta clasificación de la $R A E$ (2009: 13.2.5) que distingue entre los adjetivos de nivel individual, llamados también inherentes, caracterizadores o imperfectivos y los adjetivos llamados episódicos, de estadio o perfectivos. Los primeros atribuyen a las entidades designadas por el sustantivo ciertos rasgos inherentes, estables o consustanciales a ellas. Por el contrario, los adjetivos episódicos se refieren a estados accidentales, por lo general resultantes de algún cambio.

5 Partimos en este trabajo de la clasificación de Vañó Cerdà (1982) que diferencia entre adjetivos estativos y de sentido activo, según el modo como estos afectan a la entidad. Los adjetivos estativos (alto, fuerte) indican cualidades, estados o situaciones de índole fisica o material. Frente a estos, los adjetivos de sentido activo (listo, cariñoso, valiente) se atribuyen a un sujeto animado visto como fuente de actividad, de un comportamiento, de una forma de proceder o actuar ocasional o habitual. La clasificación podría completarse con otros trabajos sobre la clasificación adjetival, como el de Demonte (1999).
} 


\section{ESTUDIO DE PONERSE}

La construcción pronominal de poner, de la que nos ocupamos en este trabajo $^{6}$, está constituida por un objeto de cambio (A1), que actúa como sujeto, y el estado producido por el cambio (A3), que funciona como complemento predicativo:

Jorge (A1) se ha puesto enfermo (A3).

Mediante la construcción con ponerse no es posible expresar cambio de entidad - reservado a otros verbos, como hacerse-, sino solo cambio de estado; esto es, la entidad que sufre el cambio sigue siendo ella misma después de sufrirlo, de ahí que no sean viables las siguientes construcciones:

El renacuajo \{*se puso / se hizo\} rana.

Dios $\left\{{ }^{*}\right.$ se puso / se hizo\} hombre.

Ponerse permite expresar el devenir de un acontecimiento como un estado alcanzado o, lo que es igual, el inicio de ese estado por parte de la entidad que funciona como sujeto, estado que se expresa mediante el predicativo con que se combina. Así, en un ejemplo como el siguiente,

Yo tengo derecho a ser feliz y Francine se puso gorda, no tiene comparación con Jovita (Cristo, Cela, 1988: 79).

La entidad sujeto (Francine) ha sufrido un cambio con respecto a su estado físico, pues ha pasado de no estar gorda (estado A) a estarlo (estado B). El estado alcanzado se expresa mediante el adjetivo predicativo que acompaña al verbo (gorda).

Ponerse permite, asimismo, expresar la adquisición de una cualidad o característica con motivo de una conducta ocasional. Se utiliza, entonces, para indicar la atribución de una característica, propiedad o cualidad que no es propia del sujeto:

Enseguida se puso muy zalamera con Rosa y le pidió que la acompañase (Fuego, Mendicutti, 1995: 127).

En esta construcción, a la entidad que funciona como sujeto no se le atribuye una cualidad propia o definitoria, sino que se trata de una característica que sirve para calificar a dicho ente con motivo de su comportamiento, limitado en el tiempo y solo posible o válido en esa situación ocasional y temporal.

\footnotetext{
6 Véase Conde Noguerol (2013) para un estudio del verbo poner como verbo de cambio en
} construcción transitiva causativa. 
Dicho esto, decir que alguien se ha puesto zalamero implica que esa persona ha pasado a comportarse de tal modo, pero se trata de un comportamiento temporal. En este sentido, el verbo establece una oposición con el verbo hacerse que, en español, presenta el significado aspectual de cambio hacia un comportamiento duradero, definitorio, indefinido o permanente. Establecemos, así, una oposición entre un cambio hacia lo temporal-ocasional (ponerse) frente a lo permanente-definitorio (hacerse): Julia se hizo zalamera / Julia se puso zalamera. Puede establecerse, entonces, una relación entre ponerse, que se comporta como el incoativo de estar, y hacerse, como el incoativo de ser. Lo vemos reflejado en el siguiente esquema:

Ponerse + cualidad $=$ 'manifestarse de un determinado modo' (ESTAR) Hacerse + cualidad $=$ 'adquirir esa cualidad' $(S E R)$

Por otro lado, para atender a los distintos tipos de cambio que pueden llegar a expresarse con ponerse, es necesario establecer una doble estructuración dependiendo del tipo de entidad a la que el cambio afecta: distinguimos, entonces, entre objetos animados y objetos inanimados. En segundo lugar, debemos fijarnos en la naturaleza del predicativo con que se combina este verbo, pudiendo estructurar, de este modo, el cambio según afecte al comportamiento, a la forma física o al estado psíquico de un ser animado, o, por otra parte, al color, a la temperatura, al tamaño, etc., de una entidad inanimada.

En este trabajo nos ocuparemos solamente del estudio de ponerse con objetos afectados animados, dejando para otros trabajos el cambio que afecta a objetos inanimados.

\subsection{EL CAMBIO EN ENTIDADES ANIMADAS}

Cuando la entidad objeto de cambio tiene carácter animado, la construcción de ponerse + predicativo puede indicar cambio hacia un nuevo estado relativo al color, a la forma física o a la salud, pero, también, cambio en el estado de ánimo y el comportamiento. De ahí que ponerse se combine con grupos de adjetivos que implican atribuciones de tipo fisico (salud, color de piel), psíquico (estado de ánimo, sentimientos) y de comportamiento.

\subsubsection{Cambio de estado físico}

Cuando ponerse se combina con grupos de adjetivos que indican <estados de salud $>,<$ forma física $>\mathrm{y}<$ color $>7$, sirve para expresar el principio del resultado del cambio entendido como un nuevo estado de salud, una forma física determinada o un color que se conciben como estados temporales.

7 Muchos de estos adjetivos no admiten la combinación con los otros verbos: *Se quedó feo / guapo; Se hizo *blanco/ *colorado; Se hizo *sano/ *enfermo. 
Los nuevos estados adquiridos no requieren la actividad de un sujeto dinámico, sino que se trata de cambios de índole física o fisiológica en los que el rasgo [+ esfuerzo] en la consecución del cambio no tiene cabida.

\subsubsection{ESTADO DE SALUd}

Por lo general, cuando el cambio afecta al estado de salud, ponerse sirve para expresar que el objeto del cambio pasa de un estado bueno, normal u óptimo a un estado peor, por lo que los adjetivos con que se combina en su mayoría están marcados negativamente. No hemos encontrado registros de adjetivos o participios no marcados o marcados positivamente, como, por ejemplo, sano o curado (Juan se puso *sano/ ${ }^{*}$ curado). Para los cambios de estado que impliquen el paso hacia un estado de salud positivo, la lengua posee verbos plenos como curar/se o sanar/se $e^{8}$.

La explicación a la inexistencia de adjetivos no marcados semánticamente como sano o saludable + el verbo ponerse podría venir dada porque ponerse + adjetivos negativos como enfermo, grave, fatal... implica un cambio involuntario hacia un 'estado alterado', temporal, en relación con las circunstancias que podemos llamar normales de la persona. Dicho de otro modo, una persona normalmente es o está sana, de ahí que la alteración o cambio se produzca cuando ésta pasa de estar sana a enferma.

Hemos ya señalado que ponerse es un verbo incoativo, por lo que hace referencia al comienzo del estado adquirido y debemos señalar, asimismo, que se trata de un verbo puntual que, por tanto, admite complementos como súbitamente o de repente $\mathrm{y}$, naturalmente, rechaza complementos de duración, que corresponderian al estado resultado. De ahí que podamos decir

El sacerdote contó entonces que aquel peregrino, que habia salido de su tierra con otros treinta, se puso de repente enfermo cerca de Roncesvalles (El peregrino, Torbado, 1993, s/p).

y no sea posible una construcción como

*Juan se ha puesto enfermo durante diez semanas.

Si Juan ha estado enfermo durante diez semanas, utilizamos el verbo estar:

Juan ha estado enfermo durante diez semanas.

8 Sí se registra, sin embargo, el uso de ponerse con adverbios de signo positivo, como bien o mejor (Juan se puso bien/mejor) o el adjetivo bueno (A ver si te pones bueno pronto). En estos casos podemos establecer una equivalencia entre curarse o sanarse y ponerse bueno o ponerse bien, pero no entre curarse o sanarse y ponerse mejor, ya que curarse, por ejemplo, sí implicaría ponerse mejor, pero no al revés, ya que alguien puede ponerse mejor y no por ello curarse. 
Esto es así porque estar es cursivo o durativo - frente a ponerse que es puntual-, circunstancia que lo incapacita para admitir complementos de duración. Sin embargo, sí sería posible decir Se puso enfermo por unos momentos (o por un tiempo), esto es, con el complemento con por, que, en este caso, no expresa, obviamente, la duración del cambio, sino del resultado. Ponerse, en este caso, se comporta como un verbo de proyección resultativa (Porto Dapena: 1991-1992: 246).

Como se aprecia, aquí lo que se cuantifica no es la duración del estado resultante del cambio, ni tampoco el cambio, sino el lapso comprendido entre el estado sano y el estado enfermo. Notemos, no obstante, que el paso de sano a enfermo puede verse también en una perspectiva ralentizada. En este caso tiene carácter durativo porque por el umbral del cambio puede pasarse gradualmente:

Se fue poniendo enfermo poco a poco.

Así pues, nos vemos en condiciones de afirmar que las construcciones con ponerse solo focalizan semánticamente el inicio del estado adquirido, y no su duración. Asimismo, lo que lingüísticamente se codifica es el nuevo estado adquirido mediante el adjetivo atributo, que alude a una característica en relación con la cual el sujeto puede cambiar nuevamente.

Es llamativo, a su vez, el hecho de que no hayamos encontrado estos mismos adjetivos de <salud $>$ combinados con otros verbos gramaticales de cambio:

Se \{*volvió / *hizo / *quedó\} *sano / *enfermo / "grave.

Creemos que no se utilizan estas construcciones porque ponerse es el único verbo que posibilita la expresión del cambio hacia estados transitorios. Sí es posible pensar, por ejemplo, en una construcción como Se volvió un enfermo, pero, en este caso, el sintagma un enfermo se interpreta ya no como un estado, sino como una tipología humana que presenta unas determinadas características, ya no tanto de salud como de comportamiento. La entidad que sufre el cambio pasa a pertenecer al grupo de las personas enfermas, por lo que se lo caracteriza como tal.

\subsubsection{ASPECTO FÍsico}

Otro grupo de adjetivos prototípicos del individuo que eligen ponerse es el relativo a aquellos que indican <aspecto físico>:

Por lo que se ve, igual pasa con los piojos. Se comen el insecticida y se ponen más gordos (ABC, 15/11/97).

Pero ¿por qué lloras? Anda, anda, bonita, no llores que te pones muy fea (La voz, Chacón, 2002: 240). 
Hay que señalar que mediante esta construcción no se expresan todos los cambios físicos susceptibles de darse en un ser animado, pues, por ejemplo, no hemos registrado cambios que afectan a la altura: ponerse $\left\{{ }^{*} b a j o\right\}, o$ a la pérdida de alguna cualidad fisica: ponerse $\left\{{ }^{*} \operatorname{ciego} o^{9} /{ }^{*}\right.$ minusválido $\}$. Creemos que esto es así porque ponerse implica un cambio hacia un estado reversible de salud o físico y no hacia estados irreversibles (bajo, paralitico, ciego) que pueden ser expresados mediante otros verbos semicopulativos como quedarse, o mediante verbos léxicos del tipo empequeñecer, menguar, etc. Sí se encuentran casos de ponerse en combinación con el adjetivo alto en construcciones del tipo:

Eran cuatro o seis y creo que todos me expresaron cómo has cambiado, qué fuerte y qué alto te has puesto (Un loco, Viera, 2017:13).

Frente a los adjetivos de <salud $>$ que se combinan con ponerse en signo negativo (grave, enfermo, malo), es significativo que los adjetivos de <cualidad fisica> que se combinan con ponerse presentan tanto el rasgo positivo como negativo y pueden ser, por tanto, agrupados en pares antonímicos: guapo/feo; gordo/delgado: Juan se puso \{gordo, cachas / delgado\} // Juan se puso \{feo / guapo\}.

En oposición al cambio de estado de salud, los cambios de estado físico no se producen de manera repentina. No es viable un enunciado como:

*Juan se puso \{gordo / fuerte / delgado / cachas\} en un instante / en unas horas $^{10}$;

y sí lo es un enunciado como:

Juan se puso \{enfermo / nervioso / contento\} de repente.

Esto es así ya que, para que la entidad representada por el sujeto alcance un nuevo estado físico, se necesita un proceso de duración más o menos prolongada y esto descansa en nuestro conocimiento del mundo. De ahí que la característica de [- cambio repentino] no se muestre como un rasgo lingüístico pertinente. Lo que sí nos muestra la construcción de ponerse $+<$ adjetivo de cualidad física> es el estado resultante de ese proceso.

En estos casos, el cambio puede ser codificado de forma bidireccional. Alguien que está en su peso puede pasar a estar gordo o gordísimo; o a delgado o delgadísimo. Se pueden dar los siguientes procesos, por lo que el cambio puede establecerse de forma bidireccional:

\footnotetext{
9 Sí son comunes, sin embargo, expresiones del tipo: ponerse ciego de ira o ponerse ciego con la comida.

10 Podriamos expresar, sin embargo un enunciado como Juan siempre fue delgado y de repente mira lo gordo que se puso. En estos casos, la lámina temporal atribuida a de repente puede ser más o menos gruesa dependiendo del tiempo que tomemos como relevante.
} 


$$
\begin{aligned}
& \text { Estado } A \rightarrow \text { Estado } B \\
& \text { gordísimo } \rightarrow \text { gordo } \rightarrow \text { en su peso } \rightarrow \text { delgado } \rightarrow \text { delgadísimo } \\
& \text { delgadísimo } \rightarrow \text { delgado } \rightarrow \text { en su peso } \rightarrow \text { gordo } \rightarrow \text { gordísimo }
\end{aligned}
$$

Si establecemos un eje horizontal del cambio con dos polos opuestos, que irían desde lo negativo a lo positivo, o al revés, vemos que el cambio opera lingüísticamente hacia los dos polos, el positivo (fuerte, cachas, guapo) y el negativo $($ feo, gordo):

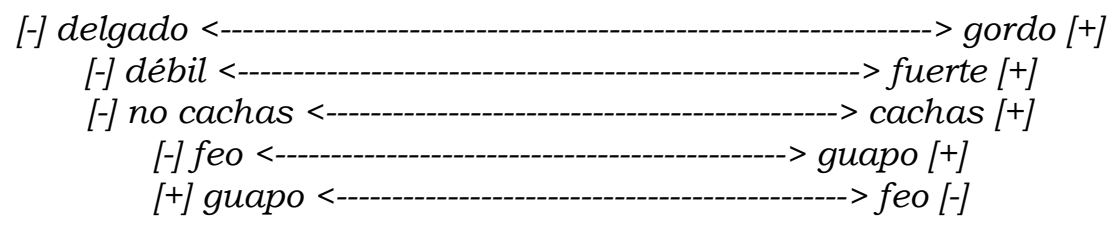

En todo caso, el uso de la lengua ha tendido al empleo de verbos deadjetivales para mostrar este tipo de cambio: adelgazar, engordar, fortalecerse, debilitarse, afearse, embellecerse. Sin embargo, aunque puedan parecer similares dos construcciones como las siguientes:

Juan engordó / Juan se puso gordo, Juan adelgazó / Juan se puso delgado, Juan se fortaleció/ Juan se puso fuerte,

existe una marcada diferencia entre unas y otras. Si decimos que alguien engordó, comparamos el nuevo estado resultante con un estado anterior, pues es evidente que con una construcción como Juan engordó implicamos que Juan está ahora más gordo que antes. Sin embargo, esto no significa que el sujeto pase a pertenecer al grupo de las personas gordas, pues es posible enunciar: Juan engordó pero sigue siendo delgado. Ahora bien, con la construcción con ponerse + adjetivo, el sujeto sí pasa a formar parte de las personas gordas, por lo que una construcción como *Juan se puso gordo pero sigue siendo delgado resulta incoherente. En este último caso lo que se compara es el nuevo estado con un estado anterior y no con una pauta.

En todos estos ejemplos el cambio es de tipo físiológico u orgánico, es decir, el sujeto no ejerce [+ control] en la adquisición del cambio, de ahí que no hayamos encontrado ejemplos de construcciones transitivas:

*Juan / la comida / la falta de ejercicio lo puso gordo.

Por otro lado, hemos comprobado que muchos de estos adjetivos referentes al aspecto físico de un individuo se combinan también con el verbo volverse: 
Juan se puso / se volvió \{gordo / feo / guapo / delgado\}.

Los adjetivos que tienen la posibilidad de combinarse tanto con ser como con estar (ser/estar feo; ser/ estar gordo; ser/ estar fuerte) presentan dos posibilidades: la de designar estados episódicos o cualidades caracterizadoras. Según el verbo semicopulativo con que se combinen actualizarán uno u otro rasgo, estableciéndose así una oposición semántica entre uno y otro verbo.

Aunque ambas construcciones, ponerse y volverse, se refieren al mismo tipo de cambio, volverse añade el rasgo [+ permanente] al estado alcanzado. Además, en una construcción como Juan se volvió feo, el atributo feo ya no se considera como un adjetivo que designa un estado episódico, sino que indica una cualidad caracterizadora que afecta la entidad que sufre el cambio. Veamos en la siguiente tabla las características de ambos verbos a que estamos haciendo referencia:

\begin{tabular}{|c|c|c|}
\hline & INCOATIVO & RESULTATIVO \\
\hline \hline -PERMANENTE & ponerse & estar \\
\hline \hline +PERMANENTE & volverse & ser \\
\hline
\end{tabular}

\subsubsection{COLOR}

El tercer grupo de adjetivos de estado físico que se combinan con ponerse es el de los que indican <color $>$.

Cuando hablamos de cambios relativos al color que afectan a entidades animadas nos referimos a la susceptibilidad que posee la piel para cambiar de color. Por lo tanto, aunque son cambios que se aplican a entidades animadas, se trata más bien de cambios que se producen en una cosa (en este caso, la piel), pero que por sinécdoque se aplican a todo el individuo:

No recuerdo la reacción de Carmen; Pilar se puso blanca (Aquellos, Feo, 1993: $\mathrm{s} / \mathrm{p})$.

Con estos adjetivos, ponerse implica también la adquisición de un estado anómalo, entendido como un nuevo color de carácter temporal (morado, colorado, blanco, pálido, etc.) con respecto al cual el objeto afectado puede nuevamente cambiar. La adquisición de estos colores por parte de la entidad sujeto son fruto de una reacción fisiológica de la piel, es decir, una reacción del cuerpo ante un estímulo o ante una determinada circunstancia externa psíquica (una emoción fuerte, un susto, una sorpresa, una situación embarazosa, etc.) o fisica (exposición al sol, una enfermedad...), por lo que el elemento [+ esfuerzo] en la consecución del cambio no tiene pertinencia. Cabe señalar que en la mayoría de los casos el adjetivo referido al color lleva implicadas también unas connotaciones psicológicas cuando se refieren a personas: ponerse blanco o pálido es algo más que cambiar de color (puede 
indicar pasar a estar nervioso, indispuesto, perplejo, etc.); ponerse colorado o rojo (puede significar tener vergüenza, etc.).

En este sentido, la adquisición o pérdida de color se produce de manera somática, de ahí que el rasgo [+ control] por parte de la persona que funciona como sujeto sea nulo.

En cuanto al tiempo externo, se deduce que el cambio de estado es repentino, por lo que son posibles construcciones del tipo:

Juan se puso rojo de repente / en segundos / instantáneamente.

Ahora bien, si pensamos en una construcción como:

Como a Juan le gustaba el vino, con los años se fue poniendo morado poco a poco,

en este caso, el cambio es paulatino y el nuevo estado alcanzado se considera permanente.

Por otro lado, la lengua permite la creación de otros verbos por el proceso de derivación y parasíntesis para referirse al cambio de color como, por ejemplo ${ }^{11}$ :

Poner blanco $\rightarrow$ blanquear, emblanquecer

Poner rojo $\rightarrow$ enrojecer, sonrojarse

Poner pálido $\rightarrow$ palidecer, empalidecer

Poner amarillo $\rightarrow$ amarillear

Poner morado $\rightarrow$ amoratarse

Debemos advertir que algunos de estos adjetivos de color son también compatibles con el verbo quedar(se):

Juan se puso / quedó \{amarillo / blanco / pálido\}.

Sin embargo, es posible establecer una diferenciación entre ambos verbos. Quedar/se expresa el resultado de un proceso truncado, mientras que ponerse, no. Dicho de otro modo, usamos quedarse cuando hay perspectiva de un nuevo cambio, pero este no se produce; con ponerse no existe esa perspectiva. Así pues, si decimos que Juan se quedó pálido es porque pensamos que ese estado podría haber desaparecido, pero permaneció, constituyendo el punto de llegada. Por otro lado, quedarse se combina solo con

\footnotetext{
${ }^{11}$ La mayoría de verbos léxicos de cambio de estado en español son verbos creados por derivación o parasintesis (enrojecer, amarillear, enfadar, entontecer, ablandar, enriquecer, afamar, oxidar, achatarrar, etc.), procedimientos que desbordan y dificultan la clasificación de los mismos debido al gran número de unidades verbales existentes. Se trata de un recurso rico y frecuente en español para la creación de verbos de cambio de estado.
} 
predicativos que expresan eventos puntuales de cambio, es decir, eventos que carecen de extensión temporal y, por tanto, de progresión. No es posible que quedarse lleve como adjunto el complemento poco a poco:

*Se quedó blanco poco a poco.

perfectamente combinable con ponerse:

Se puso blanco poco a poco ${ }^{12}$.

\subsubsection{POSTURA CORPORAL}

Se emplea también ponerse para referirse a cambios que afectan a la $<$ postura corporal>, de ahí que se combine con adjetivos del tipo tieso, derecho o recto:

Te pones tieso en el asiento y coges bien el volante. (El club, VV.AA., 2001, 132).

No hemos registrado, sin embargo, casos como los siguientes en los que aparecen otros adjetivos de postura:

Juan se ha puesto *tumbado / *sentado / *agachado / *acostado / *echado / *inclinado.

Parece que el verbo ponerse solo acepta adjetivos que permitan la intensificación:

Juan se puso muy erguido / muy derecho / muy tieso.

frente a:

Juan se puso *muy tumbado / *muy sentado / *muy agachado. que no la admiten.

Asimismo, los adjetivos incompatibles con ponerse no permiten que el sujeto sea una parte del cuerpo:

Juan puso la espalda *agachada / *sentada / *tumbada.

frente a aquellos que sí son compatibles con ponerse:

Juan puso la espalda rígida / tiesa / derecha / erguida.

12 Podría decirse, sin embargo, Se fue quedando blanco poco a poco, donde no parece haber diferencias con ponerse, por lo que podria tratarse de una neutralización semántica. 
Por otro lado, la comparación también se presenta como un mecanismo recurrente para hablar del cambio cuando este afecta al estado físico. En español, normalmente coloquial, se usan expresiones tales como ponerse como un toro / una foca / una ballena para manifestar el cambio físico que opera en una persona, la cual aumenta su grosor y/o su fortaleza, añadiendo una connotación semántica hiperbólica:

Y sobre todo Tarsis. Se puso como un toro (La torre, Arrabal, 1982, 160).

\subsubsection{Cambio de estado psíQuico}

Ponerse permite también expresar cambio de estado psíquico cuando se aplica a entidades animadas. Hemos señalado que los adjetivos relativos a la $<$ salud $>$ no requieren la actividad de un sujeto dinámico, sino que se refieren a un estado de índole física mediante el cual no se quiere caracterizar a dicho sujeto, sino indicar la situación (de salud) en la que este pasa a encontrarse. Algo parecido ocurre en el caso de los adjetivos de <estado psíquico> que también se combinan con el verbo ponerse para indicar que el objeto afectado experimenta un cambio relativo a sus facultades anímicas o emocionales; la entidad pasa a encontrarse en una situación anímica distinta de la que estaba.

\subsubsection{EsTADo ANÍmico}

El grupo de adjetivos de <estado anímico> es más numeroso que el relativo a los <estados de salud >. Algunos de los que hemos registrado son triste, contento, alegre, feliz, excitado, nervioso, cardiaco, histérico, melancólico, nostálgico, pesaroso o sombrio.

Como se comprueba, los adjetivos de estado psíquico que se combinan con ponerse son en su mayoría de signo negativo e implican también la alteración del estado anímico de la entidad a la que afectan. Sin embargo, cuando se dan parejas antonímicas (contento, feliz, alegre / triste, pesaroso, sombrio, nada contento) ambos miembros - el positivo y el negativo- admiten la combinación con este verbo:

Juan se puso \{triste / contento\}.

Asimismo, se observa que se trata de atributos que permiten gradación:

[+] muy contento-contento-nada contento [-]

[+] muy alegre-alegre-nada alegre [-]

[+] muy feliz-feliz- nada feliz [-]

[+] muy nervioso-nervioso-nada nervioso [-] 
Al igual que ocurría con los adjetivos <de salud >, - muy próximos semánticamente a estos - la sustancia semántica de los adjetivos de <estado psíquico> se relaciona con unas características sobre las cuales el sujeto es variable. Una persona puede estar triste ahora y más tarde estar contenta, o al revés, de ahí que estos adjetivos sean combinables con la cópula estar, por lo que llegan a expresar la idea de transitoriedad y reversibilidad. Veamos algunos ejemplos:

¿Se puso nervioso cuando Cruyff le comunicó que iba a jugar? - No. Estaba muy tranquilo (La Vanguardia, 02/10/95).

A la vista de los ejemplos, en los que el cambio afecta al estado de ánimo de la entidad sujeto, podemos afirmar que se trata en todos los casos de cambios hacia estados transitorios y en muchas ocasiones determinados por unas circunstancias externas (causas) que sintácticamente, en las cláusulas pronominales, se codifican como complementos circunstanciales. Los adjetivos que aparecen con ponerse y que son solo combinables con estar (excitado, triste, (loco de) contento, cardíaco) expresan la idea de transitoriedad, de ahí que se trate siempre de estados episódicos y no de cualidades caracterizadoras.

En estos contextos en los que se expresa cambio transitorio, no hemos encontrado ejemplos de ponerse + adjetivos como tranquilo, sereno, calmo... para los que ya existe un verbo específico en español, tranquilizarse, calmarse, serenarse, que implican cierta agentividad por parte de la entidad sujeto.

Podemos señalar, pues, que ponerse + adjetivos de estado psíquico implica la adquisición de un estado transitorio e involuntario, en el sentido de que no existe actividad por parte del sujeto y, por otro lado, este no adquiere ninguna cualidad.

Cabe, asimismo, la posibilidad de enunciar construcciones como:

Juan se pone histérico cada vez que te ve / cuando tiene exámenes.

que permiten hacer una lectura de estado iterativo — dada por el contexto-, que se repite cada vez que ocurren los acontecimientos indicados por el complemento adverbial (cada vez que te ve, cuando tiene exámenes...).

Asimismo, al tratarse de cambios de estado y, por ello, de estructuras eventuales complejas, se establecen dos tipos de oposiciones. En el caso de los adjetivos que implican alteración del ánimo: excitado, nervioso, histérico..., se establece una oposición entre ese estado resultante $\mathrm{B}$, que aparece explícito en la oración mediante el adjetivo (Juan se puso nervioso, Juan se puso histérico, etc.) y un estado A, anterior, que no aparece explícito en la oración y que el interlocutor infiere como opuesto al estado B (Juan se puso nervioso (estado B); antes estaba tranquilo (Estado A)). Igualmente, la opo- 
sición también puede establecerse entre un grado mayor y otro menor (anterior) de una misma característica (Juan estaba contento por la venida de su padre; pero se puso contentisimo cuando este le dio el regalo). Por otro lado, los adjetivos que denotan estados anímicos de alegría o tristeza presentan un sistema de oposición entre la ausencia o presencia de los mismos en la entidad sujeto.

\subsubsection{COMPORTAMIENTOS O DISPOSICIONES HUMANAS}

Otro grupo abundante de adjetivos que admite ponerse es el que hemos llamado de <comportamiento o disposiciones humanas>: agresivo, borde, cariñoso, chulo, pesado, violento, zalamero,etc.

Veamos algunos ejemplos que demuestran su uso:

¿Por qué cree usted que Felipe González se pone tan agresivo cuando le responde en el Parlamento? (Tiempo, 23/04/90).

Ahora son papás y Liam se pone tierno en su nuevo disco, 'Heathen chemistry' (El Pais, 05/07/04).

El cambio que opera bajo la construcción ponerse + adjetivos < de comportamiento o disposiciones humanas > es un cambio que afecta a la conducta de la entidad que funciona como sujeto, esto es, la entidad sujeto pasa a actuar o proceder de una manera determinada. En este sentido, se entiende que la entidad sujeto sufre una variación en cuanto a su comportamiento, el cual se ve afectado de forma temporal, por lo que es entendido como una manifestación ocasional.

Para mostrar un cambio en el comportamiento que se mantenga de forma indefinida la lengua ha codificado los verbos hacerse y volverse, que no parecen mostrar muchas diferencias entre ellos:

Juan \{se hizo / se volvió\} \{agresivo/zalamero/cariñoso/pesado\}.

Puede señalarse como un matiz diferenciador el hecho de que volverse parece exigir un menor control por parte del sujeto:

Juan se hizo cariñoso para estar contigo / *Juan se volvió cariñoso para estar contigo.

Se podría suponer, en todo caso, que el hablante posee una perspectiva temporal $[+\mathrm{t}]$ a la hora de utilizar hacerse o volverse, la cual es nula cuando se utiliza ponerse [-t]. Por lo tanto, diremos que los adjetivos de <comportamiento o disposiciones humanas $>$ al combinarse con ponerse designan un estado resultante que se supone transitorio con motivo de una actitud o comportamiento ocasional o puntual; y cuando se combinan con volverse o 
hacerse, designan un estado resultante indefinido, que pasa a considerarse un rasgo propio y caracterizador del individuo.

Como vemos, en este caso el verbo ponerse se combina con adjetivos de tipo activo, como agresivo, celoso o confidencial. Son adjetivos que solo se atribuyen a un sujeto animado visto como fuente de actividad, de comportamiento, de una forma de proceder o actuar. Así, decimos chistoso porque 'cuenta chistes', chulo porque 'obra con chulería', cariñoso porque 'trata con cariño', etc.

Con ponerse, podemos decir que lo que se predica del sujeto es la adquisición de una cualidad temporal con motivo de una acción que éste ha realizado, prescindiendo de si es o no de esa manera en su vida corriente y solo apoyándonos en el acto que ha realizado. En sentido amplio puede verse como un estado transitorio si se compara este comportamiento con su comportamiento habitual. Así, en una construcción como:

Juan se puso chulo con sus superiores,

inferimos que Juan, en un momento determinado y circunstancial, ha dicho determinadas frases, hecho algunos gestos y movimientos propios o relativos a la chulería; es decir, Juan ha pasado a comportarse de forma chulesca u obrado con chulería en un momento dado. El $D R A E$, en su última edición, define chulo como persona 'que habla y obra con chuleria'; ponerse chulo implicaría, entonces, la manifestación ocasional de ese comportamiento asociado a unas coordenadas espacio-temporales concretas. Ahora bien, si Juan se comporta de forma chulesca de una manera constante o regular, 'chulo' pasaría a ser un rasgo o cualidad inherente a su carácter y habría que decir, con el adjetivo sustantivado, que Juan es un chulo. De este modo se explica que no sea incoherente una secuencia como la siguiente,

Juan no es un chulo, pero ayer se puso chulo con la Benemérita,

en la que interpretamos que Juan ha actuado con chuleria ante la Guardia Civil, pero que este no es un comportamiento habitual en él.

Parece, además, que el ente que funciona como sujeto puede ejercer un cierto control sobre su comportamiento. El rasgo [+ control] se demuestra con la posibilidad de que ponerse + adjetivo $<$ de disposición humana $>$ puede aparecer con una proposición de finalidad con para:

Juan se puso cariñoso para que su padre le dejara el coche. Juan se puso agresivo para atemorizar a los ladrones.

Sin embargo, pensamos que este rasgo no es definitorio en estos casos, ya que en muchas construcciones la lengua se muestra neutral: 
Juan se puso cariñoso \{sin querer / sin darse cuenta / adrede / a propósito\}.

Como hemos visto en páginas precedentes, con otro tipo de adjetivos no era posible la presencia de estos adjuntos finales:

* Juan se puso rojo para que lo dejaran en paz.

*Juan se puso nervioso / histérico para no hacer el examen.

Los adjetivos de comportamiento que hemos registrado, como confidencial, filosófico, lírico, modoso, profesoral, reservón, superficial o trascendental implican que la entidad que funciona como sujeto se comporta de una manera relativa a lo confidente, a lo lírico, etc.; se trata de adjetivos sufijales que derivan de nombres, por lo que podemos decir, entonces, que se trata de adjetivos de tipo relacional (Demonte y Masullo 1999: 150 y ss.). Se asocia el nuevo estado alcanzado con un determinado comportamiento a través de una serie de ademanes, frases o conductas estereotipados extralingüísticamente.

Por otra parte, hemos encontrado también la presencia de determinados sintagmas preposicionales que hacen referencia a cambios que afectan a entidades animadas, mediante los cuales es posible expresar un cambio relativo al estado psíquico. Los sintagmas preposicionales que hemos registrado son del tipo en trance, de mal / buen humor, de mal talante, de mal genio, de los nervios, equivalentes en su mayoría a los adjetivos anteriormente vistos:

Algunas personas tienen su sillón favorito y si a alguien se le ocurre sentarse en él se ponen de mal humor (Feliz, VV.AA., 1986, 117).

Otras de las expresiones que se combinan con ponerse son un energúmeno / un basilisco / como una fiera / una hiena. Se trata también estados anómalos en el comportamiento de una persona. Muestran, en consecuencia, un estado de comportamiento alterado:

Finalmente descubrió lo que me traía entre manos y que entonces se puso como un basilisco (Amado, Tomeo, 1985, 9).

Muy relacionadas semánticamente con estas, las expresiones del tipo ponerse + hecho + sustantivo se emplean solo aplicadas a personas. Registramos las siguientes expresiones, todas ellas relacionadas con el comportamiento ocasional de una persona: hecho/a una furia, hecho/a una fiera, hecho/a un basilisco, hecho/a un bolo:

Usted se pone a menudo hecho un basilisco, (Articuentos, Millás, 2001, 44). 
Podemos decir que todos los sintagmas preposicionales, adverbios y construcciones son compatibles, como cabria esperar, con el verbo estar e incompatibles con ser, pues su sustancia semántica implica temporalidad:

adverbios: Juan está / *es \{bien / mal / fatal\}.

sintagmas preposicionales: Juan está/ *es \{de mal humor / de mal talante / en trance\}.

+ como + sustantivo: Juan está/ *es \{como una moto / como una ballena / como una foca?.

+ hecho + sustantivo: Juan está/ *es \{hecho una furia / un basilisco\}.

Como vemos, ponerse es compatible con el participio hecho y con otros con los que suele acompañarse: excitado, exaltado, agitado, descompuesto, crispado, encabritado. Todos estos participios pertenecen al campo de la <afección psíquica> y son indicativos también de un estado alterado por parte de la entidad sujeto:

Él se puso muy excitado mirando cómo las olas se revolvian allá abajo (Fragmentos, Martín, 1976, 120).

Se han puesto muy agitados con la autodeterminación (Tiempo, 22/01/90).

Cabe notar, sin embargo, a este respecto que en realidad estos participios están adjetivados, de ahí que admitan cuantificación: enfadado $\rightarrow$ muy enfadado, enfadadisimo; agitado $\rightarrow$ muy agitado, agitadisimo, etc.

Ahora bien, tras nuestro análisis, deducimos que ponerse no es compatible con todo tipo de participios. Pues, como vemos, no son gramaticales las oraciones siguientes:

- Con sujeto referido a cosas:

La pared se puso *pintada.

La casa se puso *construida.

La puerta se puso *abierta / *cerrada * / * rota.

El árbol se puso *caido.

La calle se puso * mojada.

- Con sujeto referido a personas:

Juan se puso \{*(muy) callado / *(muy) despistado / *despierto / *absuelto / *disgustado / *muerto / *enamorado / *ocupado / *herido / *aburrido\}.

Debido a la doble naturaleza del participio, adjetival y verbal, diremos que existen dos tipos de construcciones con el verbo estar: por un lado, las que expresan estado como característica del sujeto sin actividad: 
Juan está muy enfadado / excitado / cabreado;

y, por otro, los participios que funcionan como forma verbal y que expresan el resultado de la acción o un estado consecuencia de la acción:

Juan está \{descalzo / despierto / absuelto / muerto / enamorado / herido\}. La pared está pintada.

La casa está construida.

La puerta está \{abierta / cerrada / rota\}.

El árbol está caído.

Como se comprueba, en el primer caso, se trata de participios adjetivados y, por lo tanto, admiten cuantificación y, en el segundo caso, se trata de participios puros que no presentan tal característica.

Estos últimos ejemplos implican un desarrollo de la acción verbal que llega a un punto final después del cual no se puede continuar. La acción, en todo caso, se da como terminada y cumplida antes del tiempo que expresa el auxiliar estar. Solo en el primer caso es posible la compatibilidad con ponerse. Aun así, existen participios que indican características del sujeto y que no se combinan con ponerse:

Juan está \{disgustado / callado / ocupado / despistado\}.

Juan se puso $\left\{{ }^{*}\right.$ disgustado /* callado / *ocupado / *despistado\}.

para los que se prefiere el uso del verbo léxico: disgustarse, callarse, ocuparse y despistarse.

Pensamos, en contra de la opinión de Porroche (1990: §8.2.1), que no parece que ponerse sea compatible con participios, exceptuando los participios que muestran estado psíquico negativo y que aparecen en grado superlativo: muy excitado, excitadísimo, muy enfadado, enfadadísimo... y que, por lo tanto, se trata de un grupo muy restringido que pueden ser equiparados a adjetivos.

Además, resulta interesante constatar que ponerse presenta otra incompatibilidad categorial, pues tampoco acepta gerundios:

*Juan se puso trabajando / engordando / agravando.

*El árbol se puso cayendo / pudriendo.

Ni sintagmas nominales del tipo:

*Se ha puesto \{todo un hombre / un imprudente\}. 
Asimismo, cabe insistir en la idea de que ponerse es incompatible con adjetivos (o con cualquier tipo de predicados) que se combinen únicamente con el verbo ser.

Juan se puso \{*socialista / ${ }^{*}$ prudente / ${ }^{*}$ cuidadoso\}.

\section{Conclusiones}

Nuestra contribución al estudio de ponerse se ha basado en criterios estructurales y se ha partido de ejemplos reales de uso que han permitido obtener conclusiones acerca de su comportamiento semántico y sintáctico. A partir de las combinaciones reales de uso de este verbo con los distintos atributos hemos podido clasificar los distintos tipos de cambio que son expresables con ponerse, según afecten al estado físico — salud, aspecto físico, color y postura corporal-, al estado psiquico —estados anímicos-o al comportamiento o disposición humana.

Hemos comprobado que ponerse no permite expresar cambio de entidad pero sí el devenir de un acontecimiento como un estado alcanzado o, lo que es igual, el inicio de ese estado por parte de la entidad que funciona como sujeto, estado que se expresa mediante el predicativo con que se combina.

Ponerse ofrece la posibilidad de expresar cambios hacia estados transitorios que indican alteración de ánimo (me puse nervioso, histérico, etc.) o de color (se puso roja, pálida), los cuales, como veíamos a lo largo de las páginas precedentes, pueden establecer una relación consecuente-temporal con un factor externo que los desencadena. Ponerse expresa asimismo cambios que llevan a la entidad sujeto hacia un estado que implica un comportamiento ocasional, momentáneo o la manifestación externa de una forma de ser (se puso zalamero, se puso celoso...). En las construcciones con ponerse, a la entidad que funciona como sujeto no se le atribuye el paso hacia una cualidad propia o definitoria, sino que se trata de una característica que sirve para calificar a dicho ente con motivo de un hecho concreto, limitado en el tiempo y solo posible o válido en esa situación ocasional y temporal.

\section{REFERENCIAS}

BOSQUE, I. \& GUTIÉRREZ-REXACH, J. (2009): Fundamentos de sintaxis formal, Madrid, Akal.

CONDE NOGUEROL, M. E. (2013): Los verbos de cambio en español (tesis doctoral). Universidade da Coruña.

DEMONTE, V. \& MASULLO, P. (1999): "La predicación: los complementos predicativos", I. Bosque, I. y Demonte, V. (dirs.): Gramática descriptiva de la lengua española, Madrid: Espasa, pp. 2461-2524.

DEMONTE, V. (1999): "El adjetivo: clases y usos. La posición del adjetivo en el sintagma nominal", Bosque, I. y V. Demonte, V. (dirs.), Gramática descriptiva de la lengua española, Madrid: Espasa, pp. 129-216.

GORP, L. V. (2017): Los verbos pseudo-copulativos de cambio 
en español: estudio semánticoconceptual de hacerse, volverse, ponerse, quedarse, Madrid: Iberoamericana Vervuert, 62.

MORENO BURGOS, J. (2018): Semántica de los verbos de cambio. Un manual complementario sobre estatividad. Createspace Independent Pub.

MORIMOTO, Y. \& PAVÓN LUCERO, M. V. (2007): Los verbos pseudo-copulativos del español, Madrid: Arco/Libros.

PORROCHE BALLESTEROS, M. (1988): Ser, estar y verbos de cambio, Madrid: Arco/Libros.

PORROCHE BALLESTEROS, M. (1990): Aspectos de la atribución en español, Zaragoza: Pórtico.

PORTO DAPENA, J.-Á. (1991-1992): "Complementos de duración con por y verbos de proyección resultativa", Dicenda. Cuadernos de Filología Hispánica, 10, pp. 241-256.

REAL ACADEMIA ESPAÑOLA, (2009): Nueva gramática de la lengua española, Madrid: Espasa.

VAÑÓ CERDÀ, A. (1982): Ser y Estar + Adjetivos. Un estudio sincrónico y diacrónico, Tübinguen: Gunter Narr Verlag. 